\title{
RESULTADOS PRELIMINARES DE LA CAMPAÑA DE EXCAVACIÓN EN EL YACIMIENTO del Paleolítico medio del Barranc Fondo (Benissa, Alicante)
}

\section{ALEIX EIXEA VILANOVA @, MIGUEL ÁNGEL BEL MARTÍNEZ ํ, ÁLVARO MARTÍNEZ ALFARO @}

Dpt. Prehistòria, Arqueologia i Hª Antiga. Universitat de València. alejo.eixea@uv.es, miguel.bel@uv.es, alvaro.martinez-alfaro@uv.es

\section{ANTECEDENTES}

Son escasas las referencias que poseemos del yacimiento. Tan sólo algunas de carácter oral que se han ido transmitiendo hasta llegar a nosotros. En este sentido, debido a la falta de documentación, es el profesor de la Universitat de València, Valentín Villaverde, quien nos aportó la mayor parte de la información sobre los antecedentes históricos y arqueológicos del conjunto. El yacimiento se descubrió a finales de los años 90 cuando un grupo de personas de la zona encontró el hallazgo esporádico de algunos útiles en piedra, fundamentalmente sílex, en las inmediaciones del abrigo. Esta noticia le llegó a él mismo quien decidió realizar una primera visita para ver el estado del emplazamiento. En ésta, el mismo profesor, en un rápido vistazo de la superficie pudo encontrar una veintena de lascas de sílex y algunas raederas tanto de talla discoide como Levallois, lo que le llevaba a pensar en una cronología del Paleolítico medio. También, y a pesar de su mal estado de conservación, pudo determinar algunos restos óseos, como falanges, diáfisis y elementos articulares pertenecientes a distintos taxones como la cabra y caballo. Todos ellos también con características propias de poblaciones pleistocenas. Fue el mismo Valentín Villaverde quien lo puso en conocimiento a las autoridades del momento quienes decidieron no dar a conocer los hallazgos con el objetivo de proteger y resguardar el emplazamiento de posibles clandestinos y aficionados. Así, el yacimiento quedó en la memoria hasta que en el año 2015 el profesor Villaverde nos lo comunicó debido al interés que teníamos en continuar con la excavación de conjuntos musterienses. Fue en 2017 cuando decidimos ponernos en contacto con el alcalde y el concejal de la localidad, quienes no tenían constancia del yacimiento. Se realizó una visita y se determinó la entidad del relleno y se planificaron los trabajos a desarrollar el año siguiente solicitando el permiso de excavación a la Dirección General de Patrimonio de la Generalitat Valenciana.

\section{PRESENTACIÓN DEL YACIMIENTO}

El yacimiento del Barranc Fondo se ubica dentro del término municipal de la localidad alicantina de Benissa, a unos $70 \mathrm{~km}$ al $\mathrm{N}$ de Alicante y $110 \mathrm{~km}$ al S de Valencia (fig. 1). El abrigo, situado a unos 340 m s.n.m, se orienta 
hacia el $\mathrm{SO}$, y posee unas dimensiones máximas de $15 \mathrm{~m}$ de ancho, $8 \mathrm{~m}$ de alto y unos $6 \mathrm{~m}$ de profundidad. Geológicamente, el yacimiento reposa sobre las areniscas con glauconita, calizas arenosas y margas del Mioceno inferior (Aquitaniense superior-Burdigaliense). Esta nueva unidad manifiesta a escala de Hoja (Benissa 822, 30-32) (IGME 2008) y de región importantes cambios, tanto en sus facies como en el espesor. En ésta zona el espesor de la unidad es bastante superior al medido, siendo sus facies también diferentes y constituidas por calizas arenosas, areniscas y margas gris-azuladas, estas últimas en capas muy delgadas. Presentan fragmentos de bioclastos, de corales y moluscos, así como una gran riqueza en nódulos y concentraciones de glauconita. En otros puntos, como en la carretera de Jalón a Bernia, a la altura del P.K.8, y en contacto mecánico con el Cenomaniense, aparecen capas verticales de calizas arenosas y bioclásticas de tonos ocres y amarillentos, que alternan con niveles delgados de margas gris-azuladas, con restos de moluscos y equínidos.

La caracterización de la unidad, desde el punto de vista paleontológico, ha dado como resultado la existencia de una gran cantidad de fragmentos de algas, lamelibranquios y equínidos, así como también de foraminíferos de los grupos de las: Globigerinas, Globigerinoides, Globorotalias, Lepidocyclinas, Miogipsinas, Dentalinas y Rotálidos, que en conjunto caracterizan el Mioceno inferior, y en concreto el Aquitano-Burdigaliense. Además, esta unidad representa un brusco cambio en la sedimentación de la plataforma miocena, pasándose mediante un fuerte proceso regresivo, de facies de talud y de plataforma externa, a facies de cordón litoral.

En relación a la campaña de excavación del yacimiento, se realizó durante los días 3 al 14 de septiembre de 2018 . Se contó con la participación del equipo de dirección e investigación, formado por Aleix Eixea, Miguel Ángel Bel y Álvaro Martínez Alfaro, todos de la Universitat de València. La zona de actuación se centró, primeramente, en 3 $\mathrm{m}^{2}$ que, posteriormente y a medida que se descendía, se amplió a $6 \mathrm{~m}^{2}$. Los cuadros pertenecían a las bandas $\mathrm{C}$ y E (3 a 5) y el objetivo fue llevar a cabo una primera cata a modo de sondeo que nos permitiera ver la entidad del relleno y comprobar que el yacimiento se encontraba in situ. La zona elegida fue el lateral sagital izquierdo del abrigo en el cual se hallaba el corte a vista hecho por la maquinaria al vaciar el antiguo vertedero (fig. 2).

\section{METODOLOGÍA}

La metodología utilizada en la excavación se ha ajustado al sistema clásico de levantamiento por cuadrículas de $1 \mathrm{~m}^{2}$. Como norma general, los materiales líticos superiores a $2 \mathrm{~cm}$ de longitud y los óseos de más de $3 \mathrm{~cm}$ se posicionan tridimensionalmente, mediante el empleo de un teodolito y dibujo en papel milimetrado. Sin embargo, en el transcurso de la excavación algunos materiales identificables que no llegan a estas medidas (fragmentos

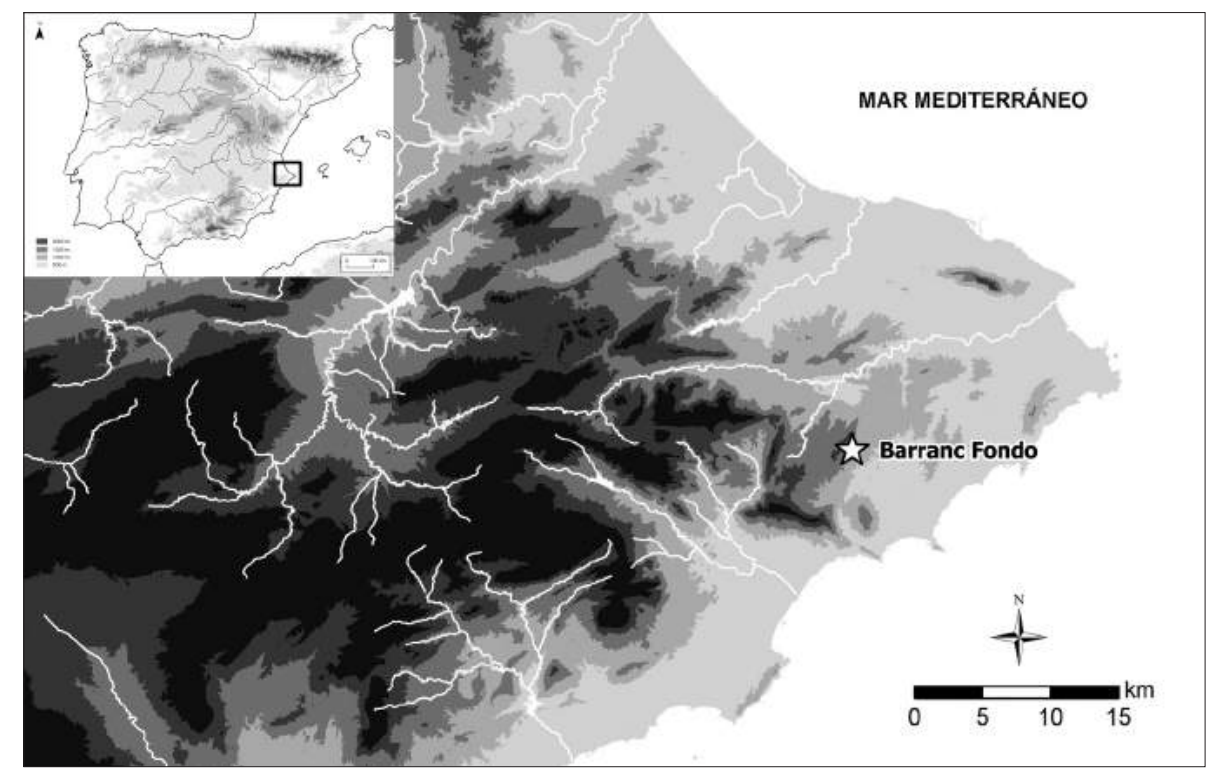

Fig. 1: Ubicación del yacimiento. 
de lascas, fragmentos retocados, epífisis, fragmentos dentales, microfauna, etc.), son también situados. Para facilitar un mayor control de la dispersión espacial del material no acotado o localizado en criba, cada metro cuadrado se divide en el proceso de excavación en subcuadros de $25 \mathrm{~cm}$ de lado $\left(0,0625 \mathrm{~m}^{2}\right)$, consiguiendo así una malla de 16 unidades por metro cuadrado.

La potencia de las tallas artificiales en las que se divide cada nivel se sitúa en torno a los $5 \mathrm{~cm}$, ajustando en lo posible el final del levantamiento a la unidad estratigráfica subyacente. El sedimento de cada subcuadro se almacena en cubos individualizados y posteriormente pasa a ser cribado. La criba se realiza en seco debido a la falta de accesibilidad de agua que hay en el yacimiento, utilizando una doble malla de 4 y $2 \mathrm{~mm}$. El material recuperado en criba, normalmente esquirlas líticas y óseas, pasa a formar parte de la bolsa de cada subcuadro para ser recontado y añadido posteriormente a la base de datos en el laboratorio.

\section{ESTRATIGRAFÍA}

Respecto a los niveles levantados, constan de un total de seis y se distribuyen de la siguiente manera (fig. 3):

- Un primer nivel I, caracterizado por una potencia desigual (entre los 5 y $10 \mathrm{~cm}$ ) y color marrón oscuro, con abundante materia orgánica (paja, elementos vegetales, excrementos de oveja, etc.), en el que no se pudieron documentar materiales arqueológicos. Tan solo algunos elementos cerámicos de época moderna.

- El nivel II, de coloración rojiza fuerte, presenta fracción pequeña y media $(3-5 \mathrm{~cm})$ de morfología redondeada. Su potencia oscila entre los 4-6 cm de espesor. El contacto con el nivel I es limpio y bien delimitado, sobre todo por su diferente coloración, aunque en algunos puntos el color oscuro del I impregna pequeñas superficies del II.

- El nivel III, similar en la composición sedimentaria que el anterior, se distingue por una tonalidad rojiza más clara e inexistencia de fracción. Sí se documenta una pequeña pulsación de unos $3 \mathrm{o} 4 \mathrm{~cm}$ de carbonatos. Su potencia es de 6-8 $\mathrm{cm}$. Sus contactos con el II y IV son complicados debido a su misma estructura arenosa y de coloración rojiza.

- El nivel IV, posee una tonalidad rojiza más oscura y tendiendo hacia colores más amarronados, no tiene fracción. Su potencia es de $5-8 \mathrm{~cm}$.

- El Nivel V se caracteriza por la presencia de numerosos grandes bloques de entre 10-20 cm que cubren la

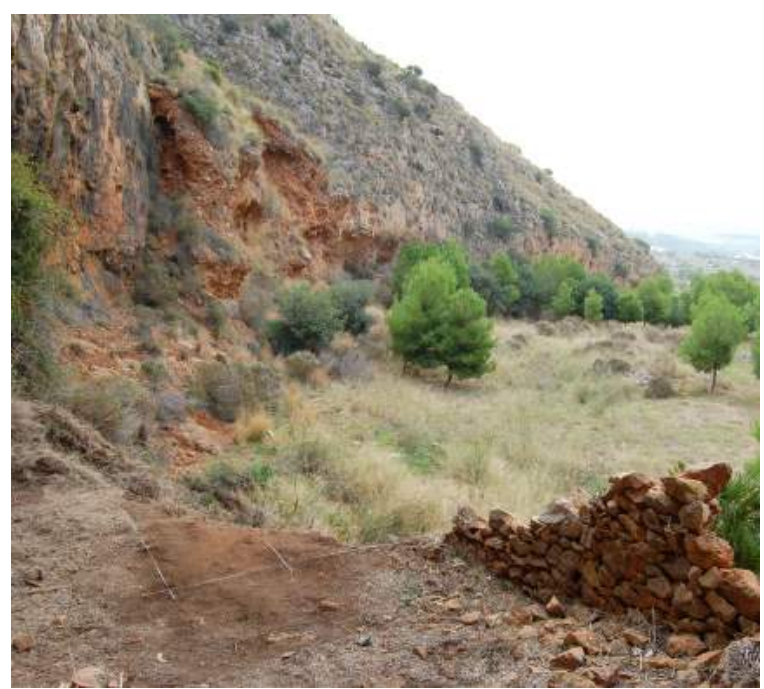

Fig. 2: Vista general de la superficie inicial de excavación.

práctica totalidad de la superficie de excavación. La sedimentación continúa siendo arenosa de tonalidad rojiza aunque un poco más clara (amarillenta). La potencia gira en torno a los 30-40 cm de espesor.

- Finalmente, el nivel VI está compuesto por una estructura de arenas, con una fracción media y grande y, todo ello, brechificado, lo que dificultó su excavación. Su potencia asciende a unos 2 metros.

\section{ESTUDIO DEL MATERIAL ARQUEOLÓGICO}

El número de elementos documentados en esta campaña de excavación es bajo, tanto a nivel lítico como óseo. Entre otros, en el primer caso, se recuperaron varios elementos líticos elaborados sobre lasca entre los que dominan un fragmento de lasca laminar y un fragmento de lasca, en ambos casos de talla indeterminada, debido a que no presentan elementos característicos propios de una gestión lítica específica (Levallois, disoide, Quina, entre otras). En ambos casos, se adscriben al nivel II. También se documentaron algunas esquirlas derivadas de la talla en el nivel III. En todos los casos, una breve observación del terreno y los análisis preliminares macroscópicos llevados a cabo, parecen apuntar hacia un tipo de sílex local que se ubica a poca distancia del yacimiento $(5-10 \mathrm{~km})$. A partir del estudio de la zona y de los mapas geológicos, se observa como durante el Cenomaniense inferior y durante todo el Turoniense, vuelve a implantarse una fuerte transgresión en la 


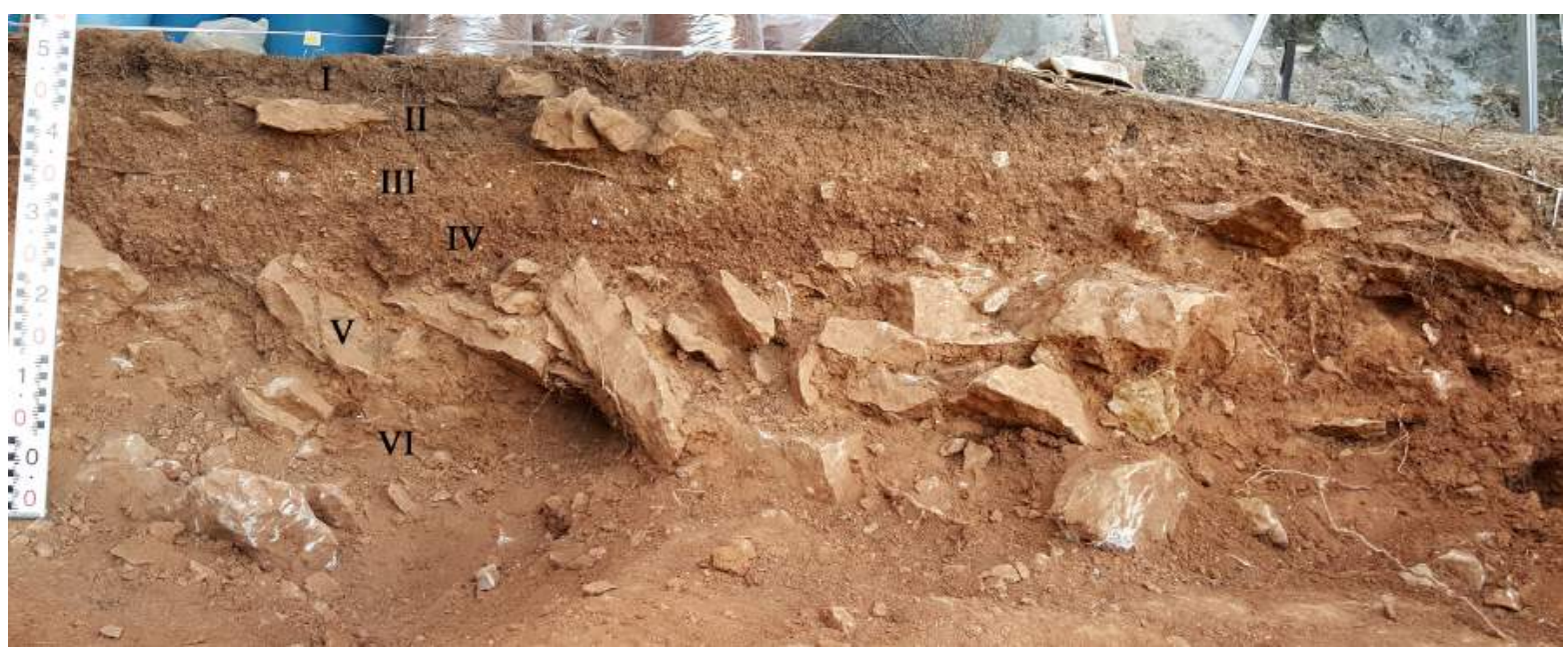

Fig. 3: Detalle del corte estratigráfico (frontal proximal).

que aparece una distribución uniforme de facies. En las partes bajas de los mismos, es dónde se depositaron potentes series de calizas con Pithonellas y nódulos de sílex (IGME 2008). La realización de nuevas prospecciones sistemáticas por el territorio circundante nos va a permitir ahondar con mayor precisión en las características de estos tipos de sílex y sus ambientes de formación.

En el segundo caso, a lo largo de todos los niveles se determinaron restos de conejo (falanges, ulnas, dientes, fragmentos de diáfisis, etc.) y, también de cabra, sobre todo a partir de elementos dentales. Excepcionalmente, en el nivel I y III se identificaron varios molares de caba1lo. Respecto a los restos indeterminados, predominan las tallas medias y pequeñas las cuales podrían corresponder a los principales taxones comentados anteriormente. En relación a la microfauna, en la separación de las tierras flotadas se han documentado a lo largo de toda la secuencia tanto elementos craneales como postcraneales los cuales se encuentran en proceso de estudio.

En todos los casos, los restos arqueológicos se encuentran poco alterados y en un buen estado de conservación (bajo índice de fracturación y fragmentación, ausencia de pátinas y termoalteraciones y muy poco concrecionados).

Finalmente, a pesar de que no se observaron hogares ni elementos de combustión (manchones cenicientos, rubefacciones, etc.), sí que se pudieron recuperar algunos restos de carbón que, a la espera de su análisis, podrán ser identificados taxonómicamente y, posteriormente, enviados a datar. Todo ello, está en manos de la Dra. Yolanda Carrión de la Universitat de València. Del mismo modo, gracias al cribado con agua de las tierras excavadas en los distintos niveles mediante el uso de máquina de flotación, se va a poder buscar en ellas elementos carpológicos que corren por parte de Carmen Martínez-Varea, también de la Universitat de València. En ambos casos, y junto con el estudio de la microfauna por parte del Dr. Pere M. Guillem, el objetivo fundamental es establecer con mayor precisión las características del conjunto desde un punto de vista cronológico y paleoambiental.

Respecto a la fauna, el análisis taxonómico y tafonómico detallado se está realizando en el Gabinet de Fauna Quaternària del Museu de Prehistòria de València por parte del Dr. Alfred Sanchis.

\section{CONCLUSIÓN}

A la luz de la intervención arqueológica llevada a cabo y con los materiales recuperados en las recogidas superficiales pasadas junto con los recuperados en la campaña de excavación, las inferencias que podemos realizar hoy en día son aún preliminares. Así y todo, podemos destacar que el emplazamiento del Barranc Fondo se presenta como un nuevo yacimiento arqueológico debido a la presencia de elementos de tipo antrópico tanto faunísticos como líticos. En este sentido, no podemos encuadrarlo cronológicamente con exactitud aunque los hallazgos conocidos con anterioridad fruto de las recogidas realizadas en los años 90 sugieren una cronología perteneciente al Paleolítico medio (industrias de lascas, elementos de talla discoide y Levallois, utillaje compuesto fundamentalmente por raederas, etc.). 
Desde un punto de vista ocupacional, la buena representación de restos faunísticos y de las partes anatómicas comentadas anteriormente, muchos de ellos de cápridos y conejos, junto con los pocos elementos antrópicos, sugiere la posibilidad de que el abrigo funcionara tanto como hábitat para pequeños carnívoros (zorros, lobos, cuones, etc.) como para los grupos humanos. Todo ello, dentro de unas pautas de ocupación del yacimiento muy esporádicas en que los aportes tanto de unos como de otros fueron muy escasos.

El estudio pormenorizado de los materiales, sobre todo los elementos faunísticos y antracológicos, esperamos que nos permita establecer con mayor precisión la cronología y la reconstrucción paleoambiental de este nuevo yacimiento y, lo que es más importante, de la zona, ya que la ausencia de este tipo de trabajos en los estudios de los yacimientos del entorno sitúa Barranc Fondo un lugar clave para la reconstrucción de las secuencias climáticas del S del País Valenciano.

\section{AGRADECIMIENTOS}

El trabajo de campo y la investigación se ha financiado a través de los siguientes proyectos de investigación: "El pasado lejano: aproximación a la conducta y la ocupación del territorio en el paleolítico valenciano" (PROMETEO/2017/060) y "Síntesis del Paleolitico medio y superior en Valencia y Murcia: aspectos cronológicos, paleoambientales, económicos y culturales" (HAR2017-85153-P). Agradecer al profesor Valentín Villaverde la ayuda prestada, del mismo modo que al Ayuntamiento de Benissa y a Benissa Impuls toda la infraestructura necesitada durante los días de excavación.

\section{BIBLIOGRAFÍA}

IGME (2008): Hoja 822, 30-32, Benissa. E. 1:50.000. Segunda serie. Primera edición. Servicio de Publicaciones. Ministerio de Industria. Madrid. 